\title{
Was wir mitnehmen sollten
}

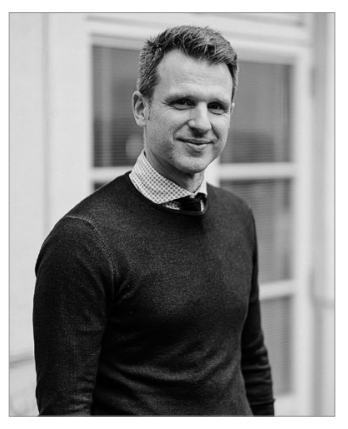

VON JOSS STEINKE

Dr. Joß Steinke ist beim Deutschen Roten Kreuz als Bereichsleiter Jugend und Wohlfahrtspflege tätig.

www.drk-wohlfahrt.de

\author{
Neben den großen Leistungen in der Corona- \\ Krise wurde auch deutlich, dass die meisten \\ Träger der Freien Wohlfahrtspflege über keine \\ solide katastrophenfeste Infrastruktur verfügen. \\ Daraus muss für die Zukunft gelernt werden.
}

Auf Twitter und im Blog der DRK-Seite www.drk-wohlfahrt.de habe ich Ende März 2020, in einer Zeit großer Unsicherheit, dazu aufgerufen, Gedanken im Sinne von "... hätten wir das mal vorher gemacht « zu notieren. Ich danke allen, die sich daran beteiligt und mich mit ihren Anregungen inspiriert haben.

Defizite und Veränderungsbedarfe sind im Zuge der Corona-Krise zutage getreten. Gleichzeitig mussten in kürzester Zeit neue Rahmenbedingungen geschaffen und Organisationsabläufe verändert werden, sodass kaum Zeit für ein Innehalten und Analysen blieb. Umso wichtiger ist es mir, jetzt einige Gedanken und Problemanzeigen festzuhalten.

\section{Wir sind "systemrelevant" "... und nun?}

Mir geht es - nicht nur in diesem Beitrag - um die Freie Wohlfahrtspflege und die Anerkennung ihrer Leistungen im Sozialstaat. Die Corona-Krise hat erneut gezeigt, wozu die Freien Träger in der Lage sind. Sie haben vor Ort die Versorgung aufrechterhalten, Unterstützung und Hilfe mobilisiert, angepackt, ausgeholfen und auf allen Ebenen in hohem Maße Präsenz gezeigt.

Dass es gelungen ist, die Entscheidungsträgerinnen und Entscheidungsträger in Politik und Verwaltung davon zu überzeugen, die Dienste der Wohlfahrtsverbände im Rettungsschirm der Bundesregierung mitzubedenken, ist das Ergebnis einer kollegialen und engen $\mathrm{Zu}$ sammenarbeit in der Bundesarbeitsge- meinschaft der Freien Wohlfahrtspflege. Gemeinsam haben wir konsequent den inhaltlichen (sozialen) Kern der Leistungen betont. Wir haben deutlich gemacht, was den Menschen fehlen würde, wenn Kinder- und Jugendhilfe, Behindertenhilfe, Beratung vulnerabler Gruppen, Mobilitätshilfen, Altenhilfe sowie die Mobilisierung der vielen Ehrenamtlichen substanziell gefährdet würden.

Sicher, wir sind in der Gesamtheit der Verbände und ihrer Gliederungen ein großer Arbeitgeber und wirtschaftlich bedeutsam, aber das spielte hier keine große Rolle. Denn die Freie Wohlfahrtspflege ist im Kern eben nicht Sozialwirtschaft und findet in Politik und Öffentlichkeit eher wenig Beachtung, wenn sie sich als ein Wirtschaftsakteur präsentiert.

Geht es um Gesellschaft und Zusammenhalt und die dafür notwendigen Strukturen, sieht das anders aus. Systemrelevanz war das Schlagwort der Stunde, und mit dem Begriff ist die Freie Wohlfahrtspflege durchaus belegt worden - gemeint waren insbesondere die Pflege- und Rettungskräfte, die Mitarbeitenden in der Eingliederungshilfe u. v. m.

Dieses Verständnis von Wohlfahrtspflege gilt es künftig weiter zu betonen und zu fördern. Dann werden wir ausreichend Rückhalt in Politik und Gesellschaft mobilisieren können. Wenn die Wohlfahrtsverbände sich auf ihre Kernkompetenz besinnen und aufhören, sich als eine Wirtschaftssparte unter vielen darzustellen, dann können sie auch in einem neuen Arrangement für eine 
zukunftsfeste und resiliente Gesellschaft ein Stützpfeiler sein. Die Mitarbeitenden haben sich in dieser schwierigen Zeit bewährt, haben ihre Kompetenz und Flexibilität bewiesen. Dies ist angesichts des hohen Misstrauens, das insbesondere der Sozialen Arbeit immer wieder entgegengebracht wird, eine große Chance.

Gleichzeitig wurde in der CoronaKrise deutlich, dass wir über keine solide katastrophenfeste Infrastruktur verfügen. Fehlende Pandemiepläne und nirgendwo ausreichende Bevorratung von Schutzmaterialien sind nur ein Ausdruck davon. Der Mangel an eingespielten ar- kein Geheimnis, dass sich die Ideengeberinnen und Ideengeber sehr schnell bei den Verbänden der Wohlfahrtspflege meldeten, um umsetzungsreife Ansätze zu generieren.

Marketing und Inszenierung sind heute Kernaufgaben, um politisch erfolgreich zu sein. Hier haben wir als Wohlfahrtsverbände möglicherweise noch Potenzial. Denn es sollte stärker deutlich werden, dass substanzielle und unverzichtbare sowie innovative Arbeit in den Verbänden geleistet wird.

Das Ringen um Anerkennung und Stellenwert könnte der Auftakt einer

\section{"Freie Wohlfahrtspflege ist im Kern nicht Sozialwirtschaft, sondern systemrelevante Daseinsvorsorge"}

beitsfeld- und organisationsübergreifenden Kommunikationswegen sowie konkreten Handlungsleitlinien ist ein weiterer Aspekt. Hier gilt es, Konzepte zu erarbeiten, Vorschläge zu unterbreiten und nicht zuletzt staatliche Investitionen einzufordern.

Dass im Zuge der Gesetzesauslegung teilweise um die Rettung von Einrichtungen auf kommunaler Ebene und um die Refinanzierung von Prämien für Pflegekräfte gerungen wurde, hat verdeutlicht, dass ein neues Arrangement zwischen Staat, Markt und den gesellschaftlich notwendigen Dienstleistungen notwendig ist.

Die frei-gemeinnützigen Träger stabilisieren und entwickeln den Sozialstaat und mobilisieren zudem in hohem Maße bürgerschaftliches Engagement, das sich auch in der Krise bewährt. In keinem Fall sind die Wohlfahrtsverbände einfach Träger von Leistungen, deren Inhalte, Umfang und Kosten von staatlichen Institutionen bestimmt werden. Und sie sollten nicht hinnehmen, weiter so degradiert zu werden.

Die Corona-Krise hat - wie im Übrigen auch schon die Lage im Zusammenhang mit der Zuwanderung Geflüchteter im Jahr 2015 - die Unverzichtbarkeit einer handlungsfähigen Wohlfahrtspflege demonstriert. Zwar musste man aufgrund der auffälligen Inszenierung zeitweise den Eindruck gewinnen, die Krise würde im Hackathon »WirVsVirus« maßgeblich bekämpft. Es ist jedoch durchaus fälligen Wohlfahrtsstaatsdebatte sein. Und zu dieser Debatte gehört ein selbstbewusstes Eintreten für Veränderungsprozesse, deren Notwendigkeit im Zuge der Krise ebenfalls deutlich wurde.

\section{Neue Herausforderungen für die Wohlfahrtspflege}

Die meisten der Rückmeldungen auf meine eingangs erwähnte Umfrage bezogen sich auf grundlegende gesellschaftliche Veränderungen. Tatsächlich hat die Krise selbst zu politischen Fragen geführt, von denen einige die Wohlfahrtspflege unmittelbar berühren.

Schulen waren offenkundig nicht adäquat vorbereitet. Die weitgehende Übertragung des Lehrauftrags an Eltern hat die Disparitäten vergrößert. Der Abstand zwischen Kindern aus Akademikerhaushalten und Kindern, die in benachteiligten oder wenig bildungsaffinen Familien aufwachsen, dürfte gewachsen sein. Aufstiegsmobilität - ein Kernanliegen der Wohlfahrtspflege - sieht anders aus. Ausdruck dessen war auch die breite Forderung von Sozial- und Wohlfahrtsverbänden, die Leistungen für Empfängerinnen und Empfänger von Grundsicherungsleistungen anzuheben.

Der Stellenwert von Kindern und (ihrer) Bildung in der Gesellschaft wurde durch die Corona-Krise somit recht deutlich demonstriert. Einen klareren Indikator als die Reihenfolge der Öff- nungen nach dem Shut-Down gibt es kaum. Insofern war die Krise auch ein ernüchternder Realitätscheck zum Aufbruch in die Wissensgesellschaft. Und es ist eine Aufgabe der nächsten Jahre, hier für echte politische Veränderungen einzutreten und jenseits hektischer Innovationsversprechen den Weg in Richtung eines verantwortungsvollen Wandels zu weisen.

Die Frauenerwerbsquote ist aufgrund der Schließungen von Einrichtungen gesunken. Dienste und Einrichtungen der Freien Wohlfahrtspflege sind eben auch Eckpfeiler einer modernen und Gesellschaft. Brechen sie weg, drohen Rückschritte.

Insgesamt hat die Krise zum Teil dramatische Auswirkungen gehabt, zum Beispiel auf Menschen, die häuslicher Gewalt ausgesetzt waren, auf psychisch Erkrankte, die zu Hause auf sich gestellt waren oder die ein erhöhtes Risiko für Angstzustände, Depressionen o. Ä. haben. Geflüchtete in Unterkünften, die ohnehin eine besonders vulnerable Gruppe darstellen, wurden vielerorts besonders drastischen Maßnahmen ausgesetzt. In großen Unterkünften in Mehrbettzimmern über Wochen auszuharren, würde für jeden Menschen eine erhebliche Belastung darstellen.

Die genannten Notlagen nachzubearbeiten und für die betroffenen Menschen mehr Sicherheit und neue sichere Orte zu schaffen, wird eine zentrale Aufgabe für die nächsten Jahre sein. Einsamkeit ist in den Monaten der Krise von einem gesellschaftspolitischen Randthema zu einem Topthema geworden. Im Deutschen Roten Kreuz sind wir dabei, Modellprojekte, die gegen Einsamkeit wirken, auf den Weg zu bringen und bereits bestehende Angebote wie ehrenamtliche Besuchsdienste, Gesundheitsprogramme und Familienangebote zu stärken. Gerade Letztere haben sich in der Krise bewährt und Wege gefunden, ihre Angebote auch unter den schwierigen Bedingungen aufrecht zu halten und für die Menschen da zu sein.

Viele Menschen sind angesichts der Veränderungen durch digitalen Wandel, Klimakrise und Corona verunsichert, das zeigt sich auf den Straßen und in den sozialen Medien. Wer ist in der Lage, verlässlich und verbindlich Brücken zu bauen, Menschen zu begleiten und einen Weg durch das Dickicht neuer Anforderungen und ganz individueller Heraus- 


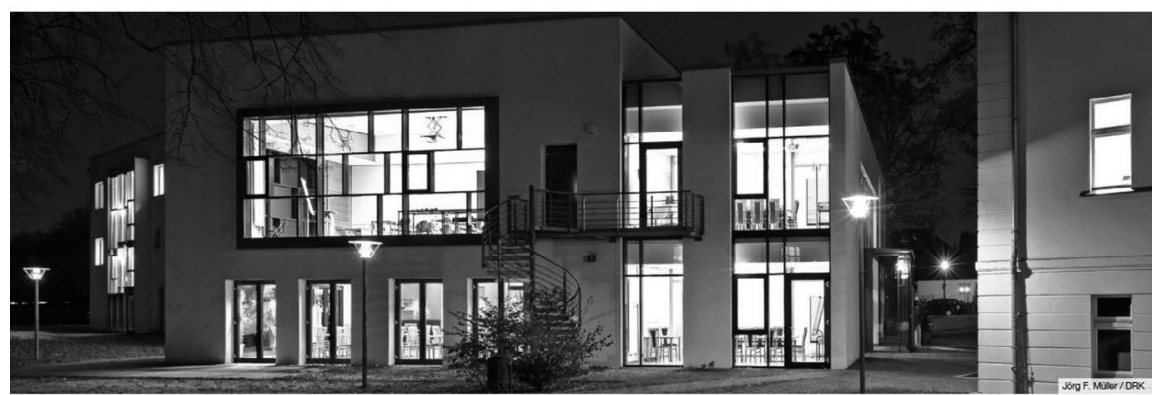

Stan / Bino

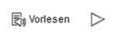

\section{Der Blog der DRK-Wohlfahrt}

In der DRK-Wohitahrt besohätrigen wir uns mit zahireiohen Fragestellungen und Themen. Für den offentliohen Dialog dazu dient dieser Blog. Wir wünsohen uns einen guten Dielog mit lhnen, für den wir Regeln in einer Nettiquette festgehalten haben. Die Beiträge stammen hauptsäohlioh Won Kolleginnen und Koll

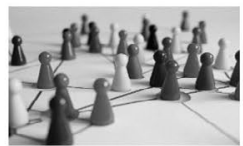

Das Netzwerk Digitale Wohlfahrt

Die digitale Transformation stellt die Wohffaht vor neue Herausforderungen. Mit dem Zel, die DRK andesverbände stäkrer zu vernetzen und gemeinsam Losungen für Heraustorderungen im Umgang mit der

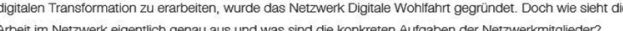
aroertim Nezzork Weiterlesen

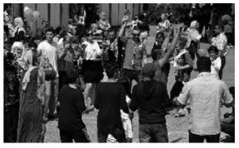

Rotes Kreuz: Eine Verpflichtung gegen Rassismus

in der aktuelen Rassismusdebatte ausgelöst durch den Tod George Floyds nach einem polizelichen Übergin meldet sich der DRK-VIzepräsident Dr. Vollkmer Schön zu Wor. Weiterlesen

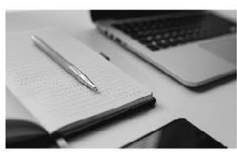

Corona-Maßnahmen der Bundesregierung: Wichtige Regelungen im Überblick [L ive Blog]

Seit Beginn der Coronaviruskise erhalten wir eine Vielzahl von Fragen zu den bundesgesetzichen Regelungen sowie Problemanzzeigen, welche Dienste und Einnichtungen der Wohtrantsppfiege noch nicht erfasst sind.

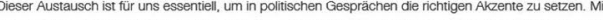
diesem Blog möchten wir Sie dabei unterstützen, die relevanten bundesgesetzlichen Regelungen für lhre Wetteriesen

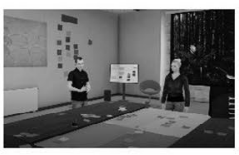

Die DRK-Digitalkonferenz in der virtuellen Realität

Da die DRK-Digitalkonferenzz nicht im geplanten Zeitraum in der realen Wett stattfinden kann, wird sie zuI Enrobung komprimierter in der virtuellen Welt stattfinden - und zwar mit vier ausgewahiten Workshops. Wie das gehen soll? In diesem Betrtrag wird vorgestellt we unsere Avatarkonterenz In den virtuellen Leern- und Arbeitswetten von TricaT funktionier

\section{$>$ Weiterlesen}

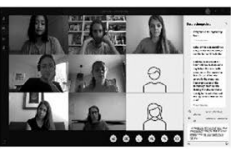

Tipps und Tricks für's Gelingen: Online Format statt

Präsenzworkshop

Wie kann man das meiste aus Onine-Events, Workshops und Webinaren herausholen? Wo liegt der Unterschied zu klassischen Präsenz-Angeboten? Diese und weitere Fragen stellen sich momentan viele. Wir in Bereich Jugend \& Wohtiaht des Generalsekketariats hatten das Gück eine Expertin hierzu befragen zu

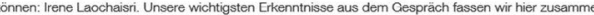
weterlesen

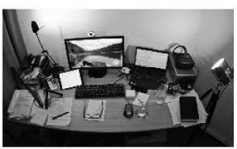

Tagungs-Talk zum Digital Social Summit 2020

Figenticich sollte der Digital Social Summit (\#DSS2020) im Mär ganz physisch in der Berfiner Kalkscheune ïber die Bühne gehen. Doch dann kam COVID-19. Wie die große Netz-Konferenz reppublica und viele andere Veranstattungen ging auch der Dioital Social Summit ins virtuelle Exil. Jennifer Geiser und Hannes Jahner waren dabei und berichten im Tagungs-Tak

$\checkmark$ Weiterlesen

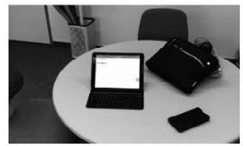

Papierfreies Büro?! - Positive Bilanz der Migrationsboratung der DRK Soziale Dienste Meschede gGmbH

Seit Juni 2019 arbeten Frau Feldhege und Frau Kotthoff als Migrationsberaterinnen der DRiK Soziale Diensto Meschede gambH an mehrereren Standdorten in Hochssauerlandkreis. Deswegenn haben sie sich für die Meschede gambH an mehreren Standorten im Hochsaverlandkreis. Deswegen haben sie sich für die Vorteile, abor auch Herausforderungen, dice es anfangs zu moistem galt.

weteresen

Der Bereich Jugend und Wohlfahrtspflege des Deutschen Roten Kreuzes konzentriert sich auf Aspekte Sozialer Arbeit innerhalb des Wohlfahrtsverbandes. In einem lebendigen Blog berichten Mitarbeitende aus allen Gliederungen über aktuelle Fragen beispielsweise von Organisationsentwicklung und Digitalisierung. Gastbeiträge sind willkommen. www.drk-wohlfahrt.de/blog/ forderungen zu weisen? Wer, wenn nicht Arbeiterwohlfahrt, Caritas, Diakonie, Rotes Kreuz, Parität und die Zentrale Wohlfahrtsstelle der Juden in Deutschland besitzt das Vertrauen, die Erfahrung und die Kontakte zu den Menschen, um dies zu leisten?

\section{Digitalisierung muss nicht hyper-innovativ sein}

Die Corona-Krise zeigt, an welchen Stellen wir in den nächsten Jahren dringend weiterarbeiten müssen. Wir waren auf eine solche Lage allenfalls in Teilen vorbereitet. Die Folge war und ist, dass die Verantwortlichen vor Ort improvisieren mussten und in vielen Fällen nicht so für die Menschen da sein konnten, wie es nötig gewesen wäre.

Digitalisierung bietet Chancen, Zielgruppen auch unter den Bedingungen einer Pandemie zu erreichen und die Soziale Arbeit neu aufzustellen. Der Wandel der Wohlfahrtspflege wurde durch Corona in dieser Hinsicht erheblich beschleunigt.

Gleichzeitig sind die Limitierungen offensichtlich. Neben der nun spürbar wachsenden Öffnung der Verbände und der Fachkräfte, die wir im Deutschen Roten Kreuz in den vergangenen Jahren mit regionalen digitalen Kompetenzzentren aktiv unterstützt haben, wären deutlich mehr Investitionen wichtig. Mehr jedenfalls als die sechs Millionen Euro, die der Bund über ein Programm des Bundesfamilienministeriums in zwei Jahren für digitalen Wandel in sechs Verbänden bereitgestellt hat.

Was möglich ist, das zeigt die OnlineBeratung in der Migrationsberatung für erwachsene Zuwanderer (MBE). Hier stand das Bundesamt für Migration und Flüchtlinge seit Jahren fest hinter der Idee, die Beratung neu und digital aufzustellen - und hat mit langem Atem für die Finanzierung gesorgt (vgl. SOZIALwirtschaft 5/2019, Seite 12 f.). Das war die Grundlage dafür, dass das entsprechende Online-Tool "mbeon « in den Monaten der Krise die Zahl der angeschlossenen Beratungsstellen mehr als verdoppelte und so Beratung auch in Zeiten geleistet werden konnte, als persönliche Kontakte gar nicht oder nur sehr beschränkt möglich waren.

Das Beispiel zeigt, was möglich ist, wenn man nicht mit eng umrissenen, kurz befristeten Projekten agiert, die alle 
hyper-innovativ sein müssen, sondern mit Fachkompetenz, etwas Behutsamkeit und ein wenig »trial and error «. Das gilt umso mehr, als digitale Angebote nie eins zu eins eine Abbildung der analogen Angebote sind.

Die Kommunikation selbst verändert sich, und es zahlt sich aus, diese Aufgabe in die Hände von Personal zu legen, das entsprechende Erfahrungen und Kompetenzen besitzt. Im Gegensatz zu den unzählbaren neuen, gut gemeinten Apps und Online-Plattformen, die in den Corona-Tagen entstanden sind, wird sich tungsträgern gemeinsam gearbeitet werden. Auch das ist ein Beitrag für eine neue Leistungs- und Krisenfestigkeit des Sozialstaats.

\section{Mehr Wohlfahrtspflege wagen}

Viele Menschen, auf die ich treffe, wünschen sich, dass die Krise zu neuen Arrangements führt, die von Entschleunigung durch weniger Dienstreisen und mehr Homeoffice gekennzeichnet sind. Im Deutschen Roten Kreuz machen wir uns Gedanken, wie wir unsere Gremi-

\section{"Digitalisierung wird schwierig, wenn Zuwendungsgeber auf gedruckten Abrechnungsbelegen und Dienstreise-Fahrkarten mit} Zangenabdruck bestehen"

»mbeon « auch nach der Krise weiterentwickeln und ist ein Beispiel für nachhaltige Investitionen in Digitalisierung.

Die Entwicklung neuer Angebotsformen ist nur eine Facette des Wandels. Vorteile hatten die Organisationen, die ihre Kommunikation, wo möglich, schon digital aufgestellt und die Verwaltung entsprechend umgestellt hatten. Gut auch, wenn man bereits Konzepte für mobiles Arbeiten und digitale Abstimmungsprozesse hatte. Wo dies nicht der Fall war, haben diese sich häufig in kürzester Zeit entwickelt. Und vieles davon wird danach Bestand haben.

Neue Settings zwischen Führungskräften und Mitarbeitenden auf der Basis von "remote work « werden in der Wohlfahrtspflege noch Zeit benötigen. Das liegt nicht allein an den Verbänden selbst. Die Zuwendungsgeber bestehen in »normalen Zeiten « auf Stundenzetteln, ausgedruckten Abrechnungsbelegen und Fahrkarten mit Zangenabdruck - und machen damit auch die Umstellung auf einen Krisenmodus zum Abenteuer.

Solange die Projektkontrolle bei den Mittelgebern so engmaschig bleibt, ist eine neue Arbeitswelt kaum erreichbar. An der Umstellung auf eine wirkungsorientierte Logik in der Projektsteuerung, Projektführung und Projektkontrolle, sollte mit Zuwendungsgebern und Leis- en neu aufstellen und Videokonferenzen so einsetzen, dass Ressourcen geschont und die Effizienz gesteigert werden. Der von mir geleitete Bereich Jugend und Wohlfahrtspflege verzeichnete 147 selbst organisierte Tagungen und 308 selbst organisierte Gremien im Jahr 2019. Da ist jede Menge Spielraum für Effizienzsteigerungen. Ähnliches gilt für die innerverbandliche und spitzenverbandsübergreifende Zusammenarbeit, die künftig ebenfalls effizienter gestaltet werden kann.

Die Erfahrungen in der Krise und die neuen Methoden der Zusammenarbeit könnten zu einem Zusammenrücken der Wohlfahrtspflege führen. Viele Kolleginnen und Kollegen auf der Bundesebene sind stolz auf die Leistungen der Menschen vor Ort. Neue Wege wurden beschritten, der »Hackathon CarehacktCorona « steht sinnbildlich dafür. Im März 2020 haben die engagierten Ehren- und Hauptamtlichen der Wohlfahrtspflege erlebt, was gemeinsam erreicht werden kann.

Auch für die Bundesregierung ist das ein Fingerzeig: Wer soziale Innovationen im Sinne eines Suchens nach den besten Lösungen für Gesellschaft und Menschen versteht und nicht als Hintergrundkulisse für hedonistische Lebensentwürfe, der darf vor allem mehr Wohlfahrtspflege wagen.

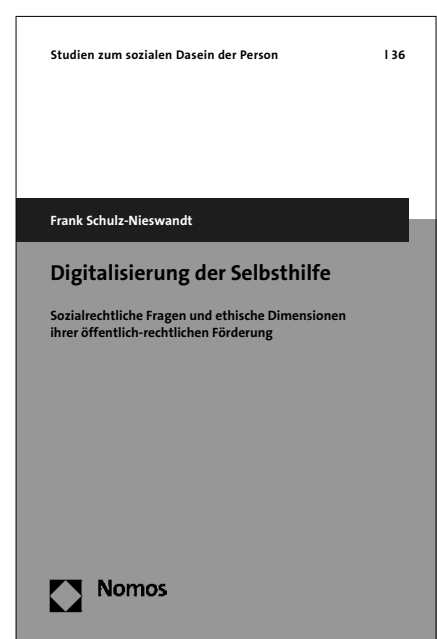

\section{Digitalisierung} der Selbsthilfe

Sozialrechtliche Fragen und ethische Dimensionen ihrer öffentlich-rechtlichen Förderung Von Prof. Dr. Frank Schulz-Nieswandt 2020, 90 S., brosch., 26,-€ ISBN 978-3-8487-6923-0

(Studien zum sozialen Dasein der Person, Bd. 36)

Die Digitalisierung verändert auch die gesundheitsbezogene Gegenseitigkeitshilfe über alle Ebenen hinweg (Verbände der Selbsthilfe, regionale Kontaktstellen, Selbsthilfegruppen) im Sinne des $\S 20$ h SGB V. Die Studie diskutiert die Auswirkungen (Chancen und Risiken), die Möglichkeiten und Grenzen einer öffentlichen Förderung digitalisierter Selbsthilfe im Sozialrecht. Dabei steht die These der Erosion der klassischen Selbsthilfegruppenbewegung im Vordergrund. Die zweite These verweist auf die Transformation der Verbände zu Service-Institutionen für Dritte als passive Konsumenten. Nicht betroffen von solchen Risiken sind die regionalen Kontaktstellen, da diese ohnehin professionelle Gebilde der Förderung von Selbsthilfeaktivitäten darstellen.

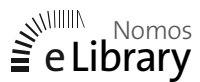

\section{Nomos}

